\title{
Simultaneous masking effect peculiar to peripheral vision
}

\author{
TAKASHI NAGANO \\ Electrotechnical Laboratory, Sakuramura, Ibarakiken, Japan
}

\begin{abstract}
In the present paper, the effects of the simultaneous presentation of a masking stimulus (an annulus) on the detectability of a test stimulus (a disk) located at the center of the masling stimulus were investigated. The results show that on the $23^{\circ}$ peripheral retina the test disk was detected more easily when presented in isolation than when presented simultaneously with an annulus, but that the effects were reversed on the fovea, where the annulus facilitated the detection of the disk.
\end{abstract}

In spite of its low visual acuity, peripheral vision is known to be indispensable for recognition in the visual world. For example, large visual objects cannot be correctly recognized with an artificially narrowed visual field even if all the parts of the objects are sequentially observed by being scanned through the use of eye movements (Ikeda \& Uchikawa, 1978; Watanabe, 1971). Information obtained by peripheral vision is considered to be an important factor in the determination of saccadic eye movements. But, in spite of the importance of peripheral vision, it has been studied far less than central vision. This is particularly true for the interaction between stimuli, although some studies in this area have been reported (Alexander, 1974a, 1974b; Bouma, 1973; Bridgeman \& Leff, 1979; Pulos, Raymond, \& Makous, 1980; Westheimer, 1965, 1967; Williams \& Lefton, 1981).

This paper describes four experiments undertaken to investigate interactions involved in the detection of one of two stimuli presented simultaneously on the peripheral retina. The two stimuli used were a disk as a test stimulus and a concentric annulus or half annulus as a masking stimulus. Simultaneous masking effects of annuli on a test spot or disk have been studied on the peripheral retina ( $7^{\circ}$ temporal), and it has been shown that the threshold of the detection of the test stimulus decreases only when test and masking stimuli are presented simultaneously (Alexander, 1974a; Pulos et al., 1980). This paper investigates simultaneous masking effects obtained at a more peripheral portion of the retina $\left(23^{\circ}\right.$ nasal) in mesopic vision.

\section{METHOD}

\section{Apparatus}

The stimuli used in these experiments were generated on the face of an oscilloscope (HP 1300A, P31 phosphor) by a minicomputer (NOVA 01). The stimuli were produced by 128 lines at a frame

The author's mailing address is: Electrotechnical Laboratory, Bionics Section, 1-1-4 Umezono, Sakuramura, Niiharigun, Ibarakiken, 305 Japan. frequency of $1 \mathrm{kHz}$, except in Experiment 3, where, because of their small sizes, the stimuli were produced by 256 lines at a frame frequency of $250 \mathrm{~Hz}$.

The stimulus field was $4^{\circ}$ in diameter and was surrounded by a large circular sheet $25^{\circ}$ in diameter and of approximately the same hue and luminance. The background luminance of the field was always kept at $17 \mathrm{~cd} / \mathrm{m}^{2}$ during the experiments.

The contrast of a stimulus, $c$, is given by $c=\left(L_{p}-L_{b}\right) / L_{b}$, where $L_{b}$ is the background luminance and $L_{p}$ is the pattern luminance. Photometric calibration showed that, for contrasts up to about 0.7 , grating contrast varied linearly with voltage.

\section{Stimuli}

The stimuli used were a bright test disk and masking stimuli. Their negatives and sizes are shown in Figure 1. In Experiments 1, 2, and 3 , the test stimulus was a disk and the masking stimuli were either full annuli or half annuli.

The percentages of correct detection of the target with and without masking stimuli were measured.
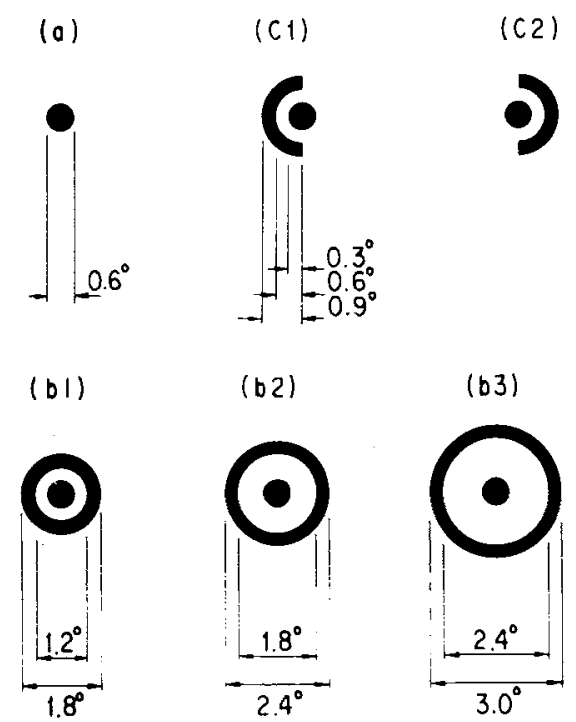

Figure 1. Stimulus patterns used in the experiments. In actual presentation, the disk, annuli, and half annuli were brighter than the background. Contrast values were chosen so that the test stimulus might be just visible. 


\section{Procedure}

The two-interval forced-choice method was used to measure the detectability of the test stimulus. When detectability of the target alone was measured, a uniform field, with a luminance of $17 \mathrm{~cd} / \mathrm{m}^{2}$, and the test stimulus, with a fixed contrast, were presented sequentially with the same exposure duration of $48 \mathrm{msec}$, as shown in Figure 2a. (No changes occurred on the stimulus field while the uniform field was being presented.) Observers were informed that the observation intervals would be marked by auditory cues. The interstimulus interval was $1.5 \mathrm{sec}$. The luminance of the field was kept at $17 \mathrm{~cd} / \mathrm{m}^{2}$ during the interval. After the second observation interval, the subjects indicated, by pushing one of two buttons, which presentation contained the test stimulus. The order of the test stimulus presentation was changed randomly on each trial. Each run consisted of 100 trials. The percentage of correct responses was calculated by using the results of 100 trials. The same procedure was used to measure the detectability of the test stimulus with a masking stimulus. In that case, target and mask durations were equal and onsets were simultaneous. The percentage of correct responses was calculated from 100 trials, each of which consisted of a sequential presentation of the test stimulus with a masking stimulus and a masking stimulus alone, as shown in Figure $2 \mathbf{b}$.

The subject sat in a chair and rested his or her head on a chinand-head brace while viewing the stimulus field. No optics or artificial pupils were used. The viewing distance was $160 \mathrm{~cm}$. Prior to initiating an experimental condition, the subject viewed the uniformly illuminated field for several minutes in order to adapt to the mean luminance of the stimulus field. The contrast values of the test stimulus and the masking stimulus were always the same in all of the experiments.

Two runs were conducted on a day. They consisted of 200 detection trials involving the test stimulus with and without a masking stimulus for a fixed contrast. The time required for each day's experiment was about $50 \mathrm{~min}$. Experiments were done at the same time each day in order to minimize any effects of day-to-day changes in sensitivity.

The data were statistically analyzed by utilizing the likelihood ratio test, in which probability density functions were approximated
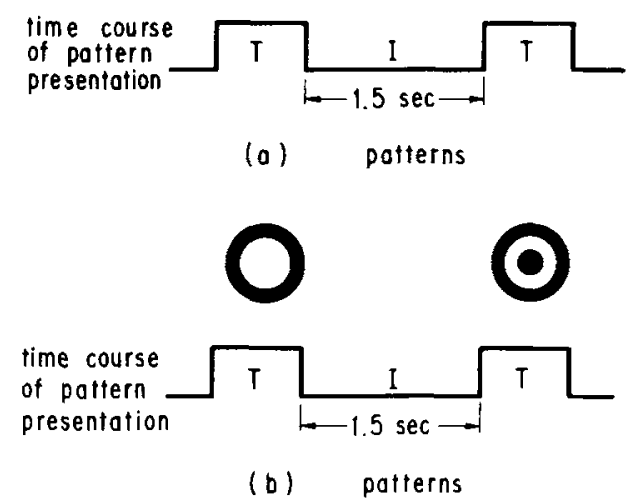

Figure 2. Ilustration for measurements with the two-interval forced-choice method. (a) The time course of pattern presentation in the case of target alone. (b) The time course of pattern presentation with a masking stimulus. The target appeared in the first or second interval randomly in each trial. Observers were informed of the occurrence of the observation intervals by an auditory cue. by normal distributions. Evaluated values of significance level shown in the results were for the least confident data in every case.

Experiment 1. This experiment was designed to determine how the detection of the test stimulus (a disk) on a peripheral part of the retina was affected by the simultaneous presentation of the masking annulus. The nasal retina $23^{\circ}$ eccentric from a foveal fixation point was chosen to represent the peripheral retina simply because its visual acuity was approximately $1 / 10$ that of the fovea.

The percentages of correct detection of the test disk were calculated from the data obtained for the stimulus conditions shown in Figures 1a, 1b1, 1b2, and $1 b 3$ and for two contrast values of the stimuli: $c=0.20$ and $c=0.25$.

To determine whether the phenomena obtained in the periphery ( $23^{\circ}$ nasal retina) would also be observed at the fovea, the percentages of correct detection of the test disk were calculated from the data obtained from the fovea for the stimulus conditions shown in Figures 1a, 1b1, 1b2, and $1 b 3$ and for two contrast values of the stimuli: $c=0.02$ and $c=0.03$. The contrast values were lower than those in the periphery because the contrast at which the test disk was just visible became lower at the fovea than in the periphery in the fixed-exposure-duration condition.

Experiment 2. Experiment 2 was undertaken to determine whether the functional differences between foveal vision and peripheral vision elucidated by Experiment 1 would hold when the stimulus sizes were chosen to be proportional to the reciprocal of visual acuity. The percentages of correct detection of the test disk were calculated from data obtained from the fovea with stimuli that had the same configurations as those in Figures $1 \mathrm{a}, 1 \mathrm{~b} 1,1 \mathrm{~b} 2$, and $1 \mathrm{~b} 3$ but were $1 / 10$ their size. This experiment was done because visual acuity is roughly 10 times higher in the fovea than in the $23^{\circ}$ peripheral retina.

Experiment 3. This experiment was designed to determine whether the finding of Experiment 1 of a masking effect peculiar to peripheral vision would occur only when the masking stimuli enclosed the test stimulus. In this experiment, the half annuli shown in Figures $1 \mathrm{cl}$ and $1 \mathrm{c} 2$ were used as masking stimuli instead of the full annuli used in Experiment 1. The contrast values were the same as those in Experiment 1.

Experiment 4. Experiment 4 examined the question of whether the disk would also suppress the detection of an annulus (or a half annulus) and hence lead to suppression of the detection of the whole stimulus. The percentages of correct detection were calculated for four patterns: the whole pattern and the full annulus in Figure lbl and the whole pattern and the half annulus in Figure 1c2. No masking stimuli were presented. The contrast values were 0.09 and 0.11 for all four patterns.

\section{RESULTS}

\section{Experiment 1}

The results of Experiment 1 are shown in Figures 3 and 4. The percentage of correct detections made in the periphery was higher when the test stimulus was presented alone than when the masking stimuli were presented at the same time $(p<.05)$. The percentage of correct detections decreased as the diameter of a masking annulus decreased. That is, the percentages of correct detections with the smallest annulus were significantly lower than those obtained with the largest one $(p<.05)$.

As for foveal vision, as shown in Figure 4, no significant differences were observed between the percentages of correct detection with and without the masking stimu- 

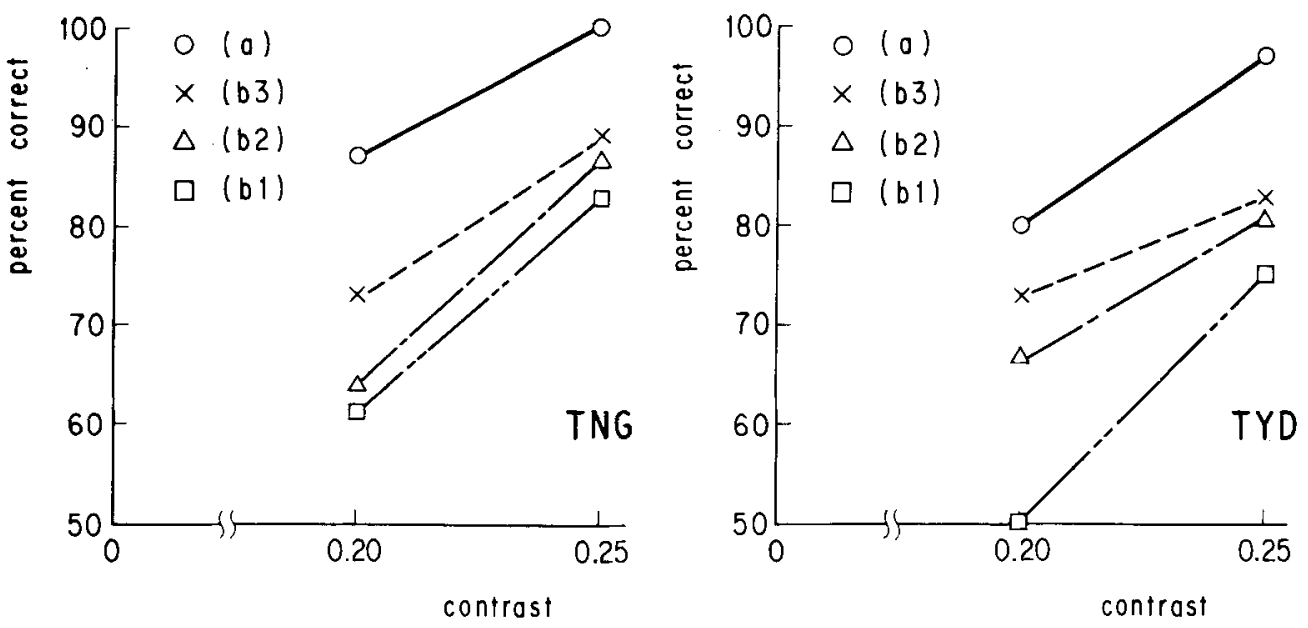

Figure 3. Effects of annuli on target detection at the nasal retina $23^{\circ}$ peripheral from the center of the fovea. The target was the disk in Figure 1a. Open circles indicate the percentage of correct detection in the case of target alone. Xs, open triangles, and open squares indicate the percentages of correct detection with masking annuli (annuli in Figures 1b3, 1b2, and 1b1, respectively).

lus $(p>.05)$, except in the case of the medium-sized annulus with $c=0.03$ in the data of T.N.G. The relationship between the diameter of the masking annulus and the detectability of the test target was not as clear as it was in the case of peripheral vision.

\section{Experiment 2}

The results of Experiment 2 are shown in Figure 5, in which it can be seen that the functional differences became clearer in this case, in that the effects of the annuli on the detection of the target in foveal vision were reversed from those in peripheral vision; that is, the masking annulus facilitated the detection of the disk $(p<.05)$ except in the cases of the smallest and medium-sized annuli with $c=0.15$ in the data of T.Y.D.

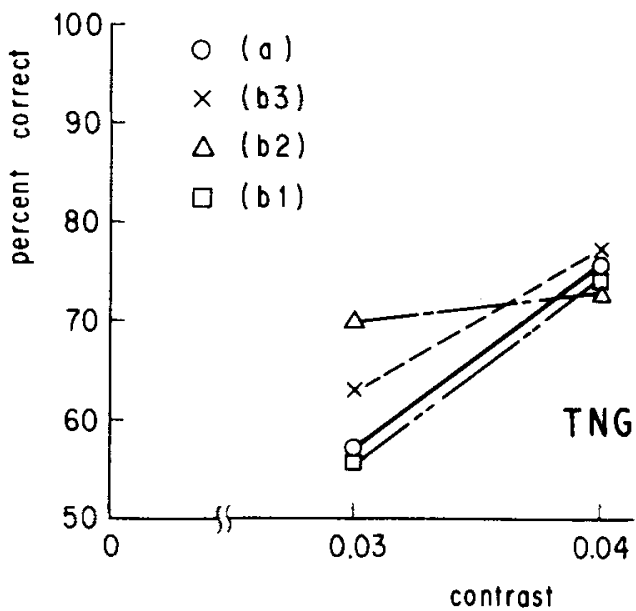

\section{Experiment 3}

The results of Experiment 3, given in Figure 6, show that it was not crucial for a masking stimulus to enclose the test stimulus. Significant differences were again observed between the cases with a masking stimulus and those without $(p<.01)$. The only requirement seems to be that the neighboring masking stimulus be larger than the test stimulus and that it be presented simultaneously with it. The results also show that the masking effect occurred regardless of whether the masking stimulus was presented on the inner or the outer side of the test stimulus. No significant differences were observed between the data obtained for the inside half annulus and data obtained for the outside half annulus $(p>.05$ ). In Figure 6, the percentages of correct detections in the case of the smallest

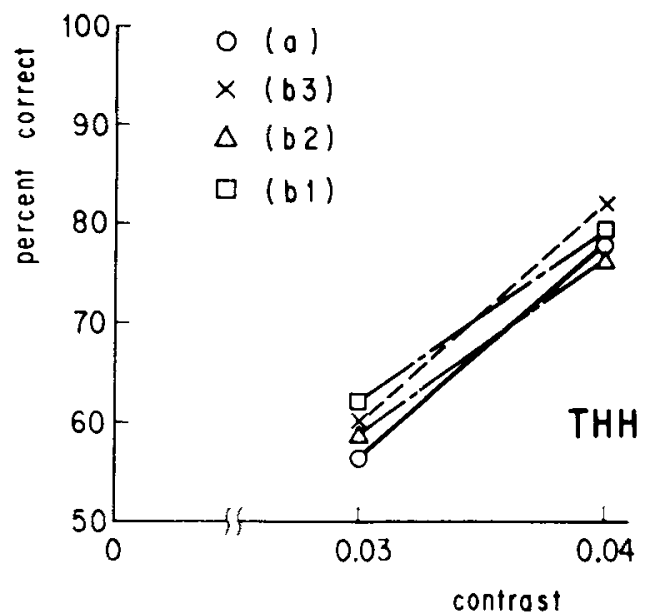

Figure 4. Effects of annuli on target detection at the fovea. The target was the disk in Figure 1a. The symbols are as for Figure 3 . 

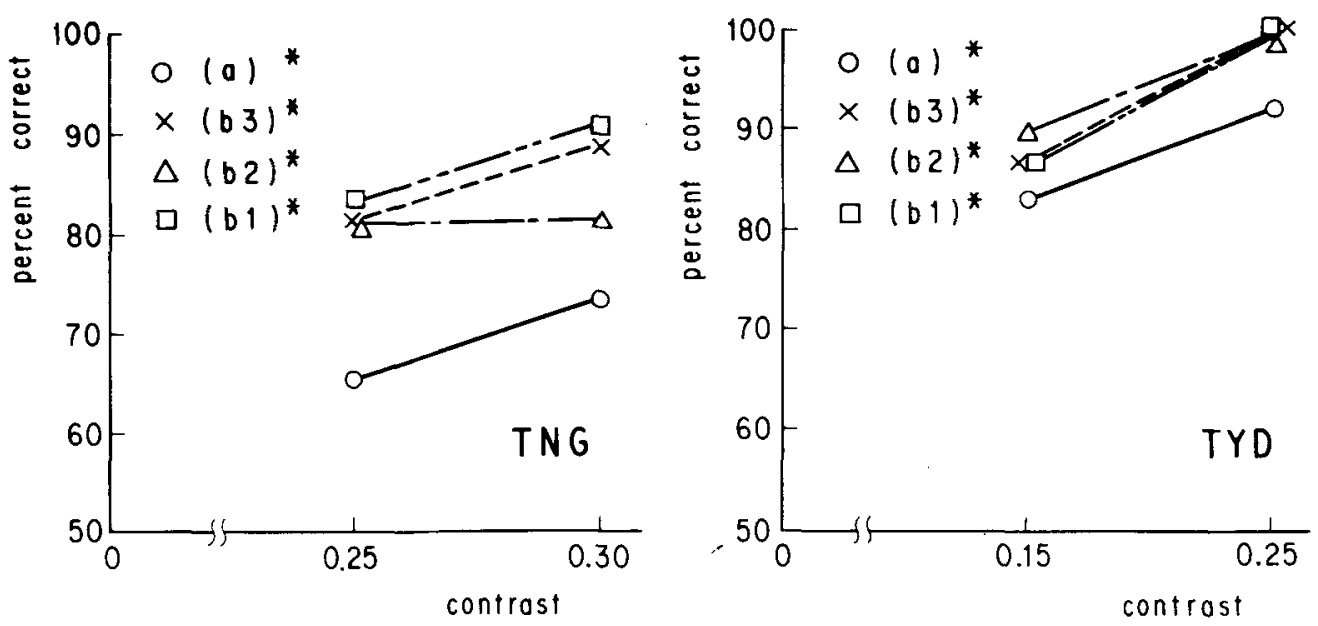

Figure 5. Effects of annuli on target detection at the fovea. The target was the disk whose size was $1 / 10$ the size of that in Figure 1a. Symbols are as for Figure 3. The asterisk means that the sizes of the stimuli used were $1 / 10$ the size of those in Figures $1 \mathrm{a}, 1 \mathrm{b1}, 1 \mathrm{b2}$, and $1 \mathrm{b3}$.

full annulus are replicated from Figure 3 so that the magnitude of the masking effect caused by a half annulus could be compared with that caused by a full annulus. Half annuli were shown to cause almost the same amount of effect as did the full annulus $(p>.05)$, except in the case of $c=0.2$ in the data of T.Y.D. This suggests that the masking effect does not linearly increase with the length of the arc of the masking stimulus but is saturated at some magnitude of the length.

\section{Experiment 4}

The results of Experiment 4 are shown in Figures 7 and 8. The detectability of the whole pattern composed of a disk and a full annulus (or a half annulus) was little different from that of an annulus (or a half annulus) alone $(p>.05)$, except in the case of the half annulus with $c=0.11$ in the data of T.N.G., where the percentage of correct detection of the whole pattern was larger than that of the half annulus $(p<.01)$. This result indicates that the suppression found is not of a mutual nature, but is a phenomenon in which the detectability of a small stimulus (fine information) is reduced by a neighboring, larger stimulus (coarse information).

\section{DISCUSSION}

The results obtained in the present study show that peripheral vision has a simultaneous masking function that is peculiar to it and hence is not observed in foveal vision. The masking effect is caused by the simultaneous
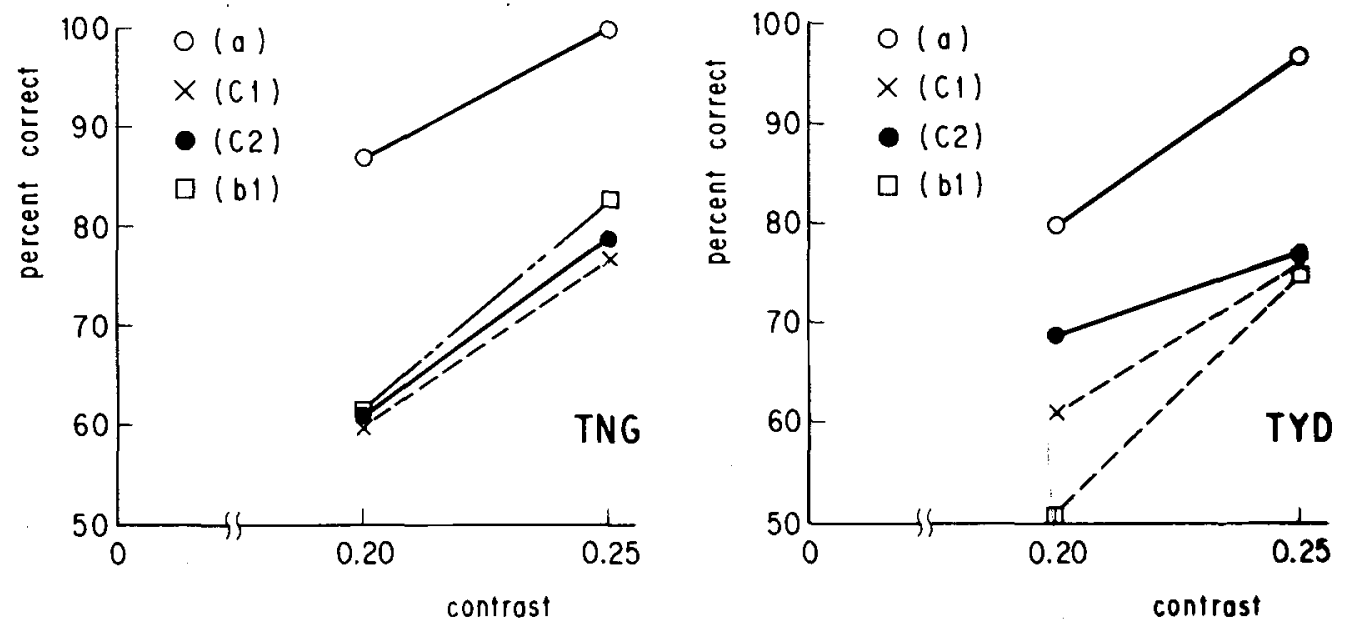

Figure 6. Effects of half annuli on target detection on the nasal retina $23^{\circ}$ peripheral from the center of the fovea. The target was the disk in Figure 1a. Open circles indicate the percentages of correct detection in the case of target alone. $\times \mathrm{s}$, solid circles, and open squares indicate the percentages of correct detection in cases with masking stimuli. a, c1, c2, and b1 indicate stimulus configurations of the target and masking stimuli shown in Figure 1. 

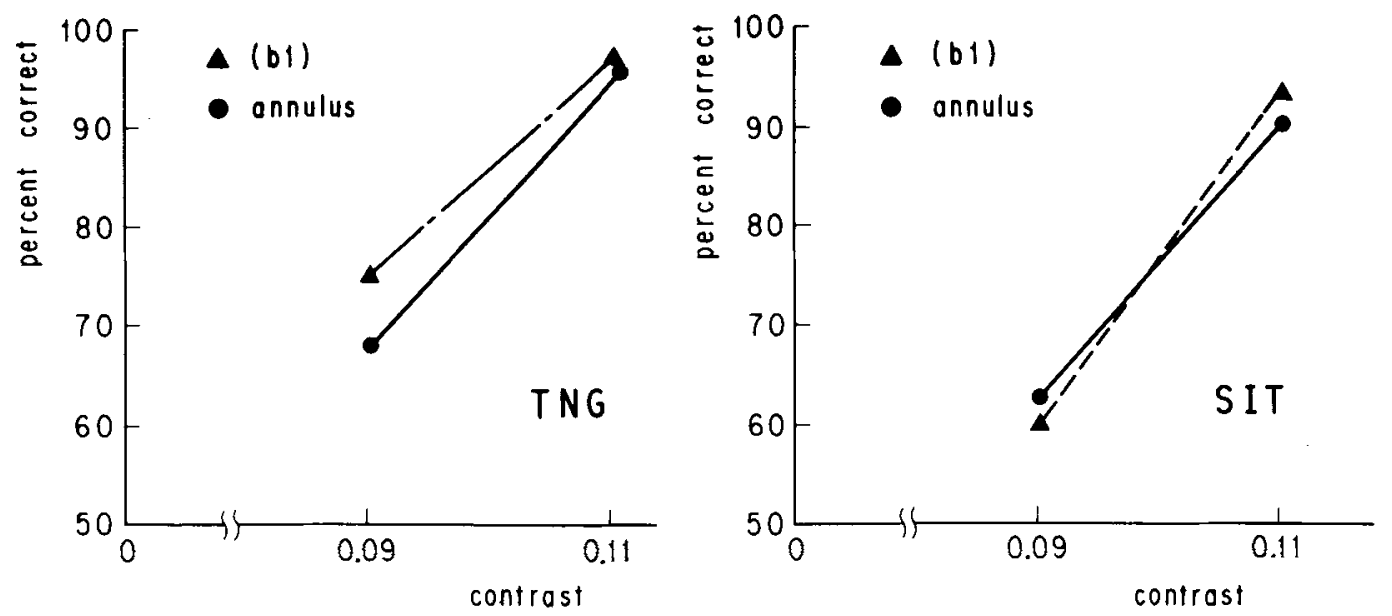

Figure 7. Effects of adding the disk to an annulus on the detection of any stimulus on the stimulus field. Solid triangles indicate the percentages of correct detection when the whole stimulus in Figure $1 \mathrm{b1}$ was presented as a test stimulus. Solid circles indicate the percentages of correct detection when the full annulus in the same figure was presented as a test stimulus. No masking stimuli were used in either case.

presentation of a masking stimulus larger in size than the test stimulus located near the test stimulus. In peripheral letter recognition, it has been shown that when two or more letters are presented simultaneously and close together, lower recognition scores are obtained because of the inhibitory interaction between them, and that this inhibitory interaction is asymmetrical and acts mainly from the peripheral side to the foveal side (Bouma, 1973; Fukuda, 1979). The masking effect found in our experiments was shown to be different in nature from this inhibitory interaction, because, as the results in Figure 6 show, this asymmetry of inhibitory interaction does not occur in detection. The magnitude of the masking effect is almost the same whether a masking stimulus is positioned on the foveal or the peripheral side of the test stimulus $(p>.05)$. Consequently, the inhibitory directional interaction results from a level of information processing that is higher than that required for detection.

The enhancement of the detection of a test stimulus has been observed in the temporal retina $7^{\circ}$ from a fixation point in the scotopic vision (Alexander, 1974a; Pulos et al., 1980). As these masking effects of annuli on a test spot or disk were dependent on the size and luminance of stimuli and the adapting field intensity (Alexander, 1974b; Pulos et al., 1980), they cannot be directly compared with the present results. But if the enhancement of detection could also occur in the mesopic vision, then the inhibition of detection would be peculiar only to the peripheral vision whose eccentricity is greater than $7^{\circ}$.

In the research of metacontrast, several relevant works on foveal vision have been reported. The detection or recognition of a test stimulus was adversely affected by
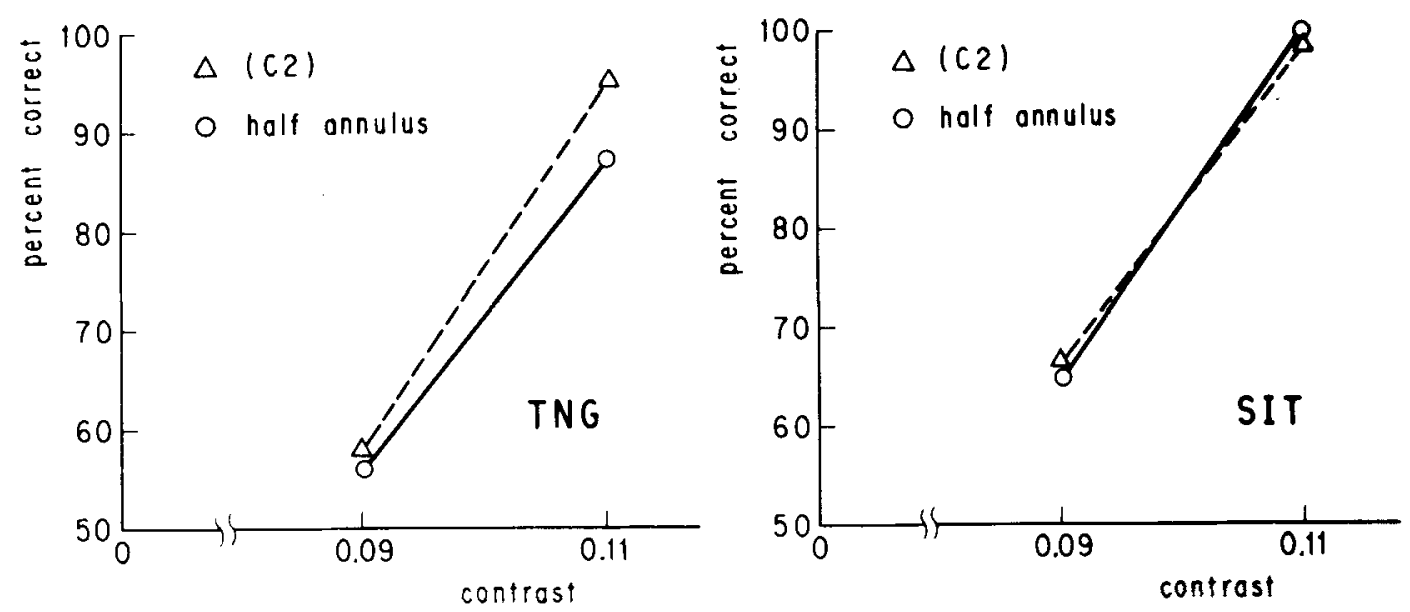

Figure 8. Effects of adding the disk to a half annulus on the detection of any stimulus on the stimulus field. Open triangles indicate the percentages of correct detection when the whole stimulus in Figure 1c2 was presented as a test stimulus. Open circles indicate the percentages of correct detection when the half annulus in the same figure was presented as a test stimulus. No masking stimuli were used in either case. 
a masking annulus when they were presented asynchronously (Eriksen \& Collins, 1964; Kolers, 1962; Stewart \& Purcell, 1970). In the case of simultaneous presentation, that is, the synchronized onsets and offsets of test and masking stimuli, two apparently conflicting results have been reported. The detection of the position of the gap in a Landolt $\mathrm{C}$ has been shown to be adversely affected by black bars placed tangential to the $\mathrm{C}$ and at a certain distance from it (Flom, Weymouth, \& Kahneman, 1963). The recognition of a single letter, on the other hand, has been found to improve when it is surrounded by an annulus in close proximity (Matthews, 1974). The results of Experiment 3 about foveal vision are consistent with Matthews's results. In both Matthews's and my experiments, very short exposure durations were used, that is, 8 to $15 \mathrm{msec}$ in Matthews's case and $48 \mathrm{msec}$ in mine. Unlimited viewing time, on the other hand, was permitted by Flom, Weymouth, and Kahneman. Since there were no other remarkable differences in the experimental conditions and stimuli used in the two works, the difference in exposure duration is thought to be the main reason for the differences obtained in the results in spite of the fact that the same simultaneous presentation condition was used.

The difference between peripheral and foveal metacontrast has also been reported by Bridgeman and Leff (1979). In some of their data, stronger masking effects were observed in the periphery than at the fovea, where few masking effects were observed. This fact is consistent with the results obtained in the present Experiment 1, even though the experimental conditions and the method of measurement were different in the two studies.

In my opinion, it would be hasty to explain the difference in terms of a model based on neural findings, although some attempts have been made to explain masking or metacontrast effects by utilizing the concept of lateral inhibition (Bridgeman, 1971; Weinstein, 1972), or sustained and transient channels (Breitmeyer \& Ganz, 1976), because psychophysical phenomena would be concerned not merely with the retina but with the total visual system. Only one thing is certain, and that is that the transient channel of the visual system would work mainly in Matthews's and my experiments and the sustained channel would work in Flom, Weymouth, and Kahneman's.

\section{REFERENCES}

AleXANDER, K. R. (1974a). Sensitization by annular surrounds: Sensitization and the contrast flash effect. Vision Research, 14, 623-631. Alexander, K. R. (1974b). Sensitization by annular surrounds: The effect of test stimulus size. Vision Research, 14, 1107-1113.

Bouma, H. (1973). Visual interference in the parafoveal recognition of initial and final letters of words. Vision Research, 13, 767-782.

BREITMEYER, B. G., \& GANZ, L. (1976). Implications of sustained and transient channels for theories of visual pattern masking, saccadic suppression, and information processing. Psychological Review, 83, 1-36.

Bridgeman, B. (1971). Metacontrast and lateral inhibition. Psychological Review, 78, 528-539.

BRIDGEMAN, B., \& LeFF, S. (1979). Interaction of stimulus size and retinal eccentricity in metacontrast masking. Journal of Experimental Psychology: Human Perception \& Performance, 5, 101-109.

ERIKSEN, C. W., Coluns, J. F. (1964). Backward masking in vision. Psychonomic Science, 1, 101-102.

Flom, M. C., Weymouth, F. W., \& Kahneman, D. (1963). Visual resolution and contour interaction. Journal of the Optical Society of America, 53, 1026-1032.

FUKUDA, T. (1979). [Visual interference in the peripheral recognition of embedded letters.] Journal of the Institute of Television Engineers, 33, 726-731. (In Japanese)

IKEDA, M., \& UCHIKAWA, K. (1978). Integrating time for visual pattern perception and a comparison with the tactile mode. Vision Research, 18, 1565-1571.

Kolers, P. A. (1962). Intensity and contour effects in visual masking. Vision Research, 2, 277-294.

MatThews, M. L. (1974). Facilitation of letter recognition by an annulus in a metacontrast paradigm. Quarterly Journal of Experimental Psychology, 26, 312-323.

Pulos, E., Raymond, J. E., \& Makous, W. (1980). Transient sensitization by a contrast flash. Vision Research, 20, 281-288.

Stewart, A. L., \& Purcell, D. G. (1970). U-shaped masking functions in visual backward masking: Effects of target configuration and retinal position. Perception \& Psychophysics, 7, 253-256.

Watanabe, A. (1971). [Fixation point and the eye movements.] Oyo Butsuri, 30, 330-334. (In Japanese)

Weinstein, N. (1972). Metacontrast. In D. Jameson \& L. M. Hurvich (Eds.), Visual psychophysics [Vol. 7, Part 4 of H. Autrum, R. Jung, W. R. Loewenstein, D. M. McKay, \& L. Teuber (Eds.), Handbook of sensory physiology]. Heidelberg: Springer.

WeSTHEIMER, G. (1965). Spatial interaction in the human retina during scotopic vision. Journal of Physiology, London, 181, 881-894.

WESTHEIMER, G. (1967). Spatial interaction in human cone vision. Journal of Physiology, London, 190, 139-154.

Williams, L. J., \& Lefton, L. A. (1981). Processing of alphabetic information presented in the fovea or the periphery: Functional visual field and cognitive load. Perception, 10, 645-650.

(Manuscript received May 12, 1985;

revision accepted for publication September 17, 1986.) 